\title{
Third order sliding mode control with a predefined convergence time: application to an electropneumatic actuator
}

\author{
Asif Chalanga and Franck Plestan
}

\begin{abstract}
This paper presents the practical implementation of a new robust third order sliding mode controller for an electropneumatic actuator with a predefined convergence time. The controllers robustness is discussed with respect to external disturbances. Implementation results on an electropneumatic actuator setup show the effectiveness of the controller.
\end{abstract}

\section{INTRODUCTION}

Pneumatic actuators are widely used in industrial applications due to the following advantages: low maintenance cost, lightweight and good force/weight ratio [1], [2], [3]. For high performance position control of electropneumatic actuators, robust control approaches are required to reduce the effect of the model non-linearity such as friction, variation of the actuators dynamics due to large change of load. Sliding mode control (SMC) is a one of the most popular robust control strategy for uncertain nonlinear systems [4], [5], [6]. The main advantages of SMC are the finite time convergence and the robustness with respect to uncertainties and perturbations. In addition to this, higher order sliding mode control (HOSM) also preserves the main advantages of SMC but improves the performance of the closed-loop system by reducing the chattering phenomenon [7], [8], [9]. In HOSM, twisting-control and super-twisting controller are widely studied [5]. Super-twisting control became more popular than twisting one due its advantages like use as observer, differentiator and generates continuous control [10], [11]. Note that these two controllers are second order sliding mode ones. They do not allow to guarantee the finite time convergence of the electropnematic actuator position, given that the system with respect to the position has a relative degree equal to 3 .

Concerning the application of SMC and HOSM to the electropneumatic system, many results have been published. A few of them are detailed in the sequel. In [12], adaptive super-twisting control is used for the piston position tracking, a linear sliding surface being used in the control design. In [13], [14], different HOSM control eventually output feedback have been used to ensure trajectories tracking. Some of recent works are also presented in [15], [16], and will be expose in reference therein. Note that these solutions can not allow to predefine the convergence time of the closed-loop system.

The main focus of this paper is the design of a new third sliding mode controller inspired from [17] for the

Asif Chalanga and Franck Plestan are with Ecole Centrale de Nantes- LS2N, UMR CNRS 6004, Nantes, France Emails: Asif-Hasanbhai.Chalanga@irccyn.ec-nantes.fr; Franck.Plestan@irccyn.ec-nantes.fr electropneumatic actuator. The controller allows to ensure the convergence of the closed-loop system in a predefined time. The dynamics of electropneumatic setup considered in the present paper are nonlinear and affected with external disturbance. After selecting proper output variable for controller design, then dynamics of the electropneumatic setup becomes similar to the system discussed in [17]. The controller objective is to force the piston position of the electropneumatic actuator to track a desired trajectory in a predefined convergence time, the time being defined in advance. Results on similar applications based on [18] have been obtained in [19], [20]. But the designed control input in [18] is discontinuous because discontinuous term appears in the control input which can cause the chattering and then affect the tracking performance. In the current paper, to reduce chattering and to improve the results of [19], a new scheme inspired from [17] and based on integral sliding mode control (ISMC) approach is used. In this scheme, discontinuous part of the control appeared in [19], [20] is replaced by supertwisting, which makes overall control continuous. This kind of robust controller, from the application point of view, is better than the discontinuous control.

\section{A. Main Contribution}

The main contribution of this paper is the design of control scheme inspired by [17] to a perturbed electropneumatic actuator. The control scheme is based on ISMC in which the "so-called" nominal control tracks the disturbance free system in a predefined convergence time. For the disturbance compensation super-twisting is used, which makes overall continuous control and improves the tracking performance.

\section{B. Structure of the Paper}

The paper is organized as follows. Section II describes the eletropneumatic system. Section III details design of the controller. Experimental results of the proposed control method to an electropneumatic system are discussed in Section IV.

\section{Description OF Electropneumatic System}

The electropneumatic system (Figure 1) is composed of two actuators ([12], [13], [14], [16], [21]): the first one, named "main actuator" and the second one that is "perturbation actuator". The main actuator composed of two chambers (denoted by $P$ and $N$ ) is a double acting actuator which is controlled by the two servo distributors. The piston diameter is $80 \mathrm{~mm}$ and rod diameter is $25 \mathrm{~mm}$. With a source pressure equal to $7 \mathrm{bar}$, the maximum force developed by the 

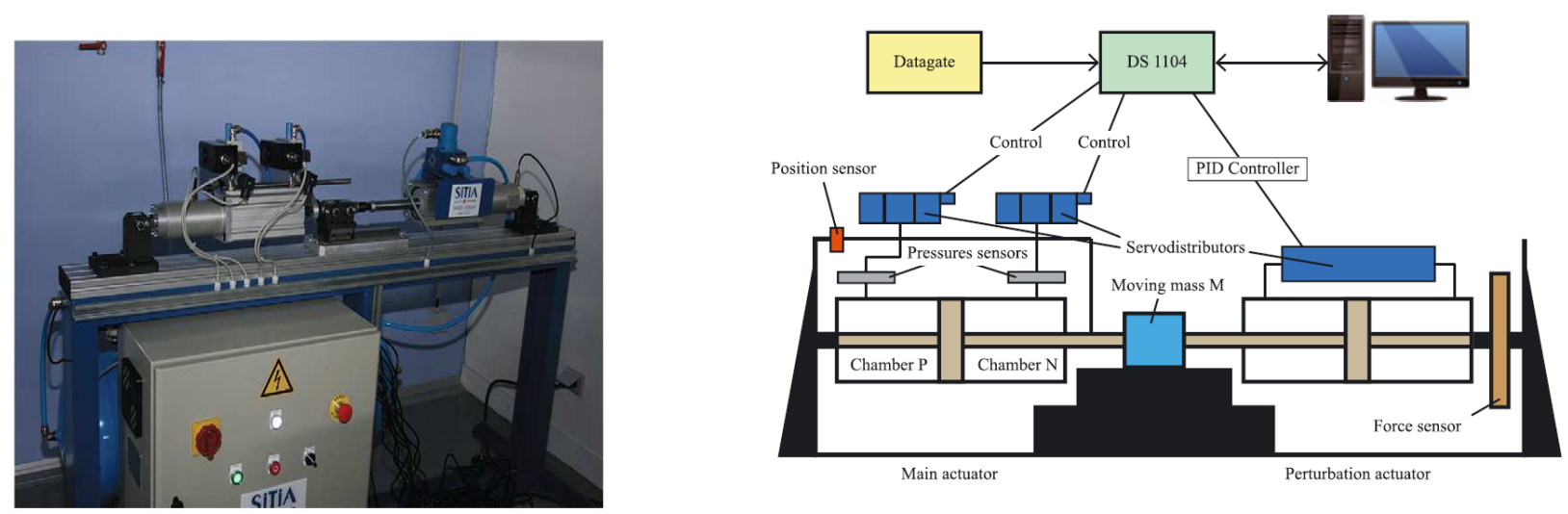

Fig. 1. Photo of the electropneumatic system and Schematic of electropneumatic system [14]

actuator is $2720 \mathrm{~N}$. The air mass flow rates entering in the chambers are modulated by two three-way servodistributors. The pneumatic jack horizontally moves a load carriage of mass $M$. This carriage is coupled with the second pneumatic actuator. The goal of the second actuator is to produce a dynamical load force on the main actuator. The second actuator has the same mechanical characteristics as main actuator, but the air mass flow rate is modulated by a single five-way servodistributor. Note that the force control of the second actuator is performed by analogic PID controller developed by the test bench manufacturer.

As detailed in [14], the nonlinear plant model of electropneumatic system is obtained from the physical laws and reads as

$$
\begin{aligned}
\dot{p}_{P} & =\frac{k r T}{V_{P}(y)}\left[\varphi_{P}+\psi_{P} \cdot u-\frac{S}{r T} p_{P} v\right] \\
\dot{p}_{N} & =\frac{k r T}{V_{N}(y)}\left[\varphi_{N}-\psi_{N} \cdot u+\frac{S}{r T} p_{N} v\right] \\
\dot{v} & =\frac{1}{M}\left[S\left(p_{P}-p_{N}\right)-b_{v} v-F_{\mathrm{ext}}\right] \\
\dot{y} & =v
\end{aligned}
$$

The nonlinear system with $\bar{z}=\left[\begin{array}{llll}p_{N} & p_{P} & v & y\end{array}\right]^{\top}$ reads as $\dot{\bar{z}}=f(\bar{z})+g(\bar{z}) u$ where

$$
\begin{aligned}
f(\bar{z})= & {\left[\begin{array}{c}
\frac{k r T}{V_{P}(y)}\left[\varphi_{P}-\frac{S}{r T} p_{P} v\right] \\
\frac{k r T}{V_{N}(y)}\left[\varphi_{N}+\frac{S}{r T} p_{N} v\right] \\
\frac{1}{M}\left[S\left(p_{P}-p_{N}\right)-b_{v} v-F_{\mathrm{ext}}\right] \\
v
\end{array}\right] } \\
g(\bar{z})= & {\left[\begin{array}{c}
\frac{k r T}{V_{P}(y)} \psi_{P} \\
\frac{k r T}{V_{N}(y)} \psi_{N} \\
0 \\
0
\end{array}\right] }
\end{aligned}
$$

The variable $y$ is piston position, $v$ is its velocity, $p_{P}$ and $p_{N}$ are the pressures in both chambers ( $P$ and $N$ chambers, respectively), $F_{\text {ext }}$ is the external force produced by the perturbation actuator, $r$ is the perfect gas constant, $b_{v}$ the viscous friction coefficient, $T$ the supply temperature, $k$ the polytropic constant and $u$ is the control input of the system. Given that the actuator is controlled by two servo-distributors through two inputs $u_{P}$ and $u_{N}$, thus $u=u_{P}=-u_{N}$. The volume in each chamber is defined as

$$
\begin{aligned}
& V_{P}(y)=V_{0}+S \cdot y \\
& V_{N}(y)=V_{0}-S \cdot y
\end{aligned}
$$

with $V_{0}$ being equal to the half of the cylinder volume and $S$ is a piston surface. The function $\varphi_{*}$ and $\psi_{*}$ with $*=$ $\{P, N\}$ are describing the mass flow rate and defined as a $5^{\text {th }}$ order polynomial [for details see [12] ]. The constraint are as follows

$$
\begin{aligned}
& |y| \leq 72 \mathrm{~mm}, \quad|v| \leq 1 \mathrm{~m} . \mathrm{s}^{-1}, \quad 1 \text { bar } \leq p_{P} \leq 7 \text { bar } \\
& 1 \text { bar } \leq p_{N} \leq 7 \text { bar, and } \quad|u| \leq 10 \mathrm{~V} .
\end{aligned}
$$

The parameters of the electropneumatic system are listed in Table I.

TABLE I

PARAMETERS OF THE ELECTROPNEUMATIC S YSTEM

\begin{tabular}{|l|l|l|}
\hline Parameters & Notation & Value \\
\hline Viscous friction coefficient & $b_{v}$ & 50 \\
\hline Polytropic constant & $k$ & 1.2 \\
\hline Perfect gas constant & $r$ & $287 \mathrm{~J} \mathrm{Kg}^{-1} \mathrm{~K}^{-1}$ \\
\hline Temperature & $T$ & $293^{\circ} \mathrm{K}=20^{\circ} \mathrm{C}$ \\
\hline Mass & $M$ & $3.4 \mathrm{Kg}$ \\
\hline Piston surface & $S$ & $0.0045 \mathrm{~m}^{2}$ \\
\hline Half-cylinder volume & $V_{0}$ & $3.40 \times 10^{-4} \mathrm{~m}^{3}$ \\
\hline
\end{tabular}

\section{Controller Design}

The objective of the control law is that the actuator position is tracking a desired position trajectory in a predefined time $t_{F}$. Define the tracking error as

$$
\sigma=y-y_{\text {ref }}, \quad \dot{\sigma}=v-\dot{y}_{\text {ref }} \quad \text { and } \quad \ddot{\sigma}=\dot{v}-\ddot{y}_{\text {ref }} .
$$


with $y_{\text {ref }}$ a sufficiently differentiable reference trajectory. The dynamics of $\ddot{\sigma}$ reads as

$$
\ddot{\sigma}=\frac{1}{M}\left[S\left(p_{P}-p_{N}\right)-b_{v} v-F_{\text {ext }}\right]-\ddot{y}_{\text {ref }}
$$

Control input is not explicitly appearing in the dynamics of $\ddot{\sigma}$. Indeed relative degree of system (1) with respect to the $\sigma$ is equals 3 , given that

$$
\begin{aligned}
\dddot{\sigma} & =\frac{1}{M}\left[S\left(\dot{p}_{P}-\dot{p}_{N}\right)-b_{v} \dot{v}-\dot{F}_{\text {ext }}\right]-\dddot{y}_{\text {ref }} \\
& =\Psi(\cdot)+\Delta(\cdot)+\Phi(\cdot) \cdot u
\end{aligned}
$$

with

$$
\begin{aligned}
\Psi(\cdot) & =\frac{S k r T}{M}\left[\frac{\varphi_{P}}{V_{P}(y)}-\frac{\varphi_{N}}{V_{N}(y)}\right]-\frac{S^{2} k v}{M}\left[\frac{p_{P}}{V_{P}(y)}+\frac{p_{N}}{V_{N}(y)}\right] \\
& -\frac{b_{v}}{M^{2}}\left[S\left(p_{P}-p_{N}\right)-b_{v} v\right]-\dddot{y}_{\mathrm{ref}} \\
\Phi(\cdot) & =\left(\frac{S k r T}{M}\left[\frac{\psi_{P}}{V_{P}(y)}+\frac{\psi_{N}}{V_{N}(y)}\right]\right) \\
\Delta & =\frac{b_{v} F_{\mathrm{ext}}}{M^{2}}-\frac{\dot{F}_{\mathrm{ext}}}{M}
\end{aligned}
$$

Note that it is assumed that the system model is known. To cancel the known parts, control input for system (3) is defined as follows,

$$
u=\frac{1}{\Phi(\cdot)}(-\Psi(\cdot)+\bar{u})
$$

After substituting the control input (4) in (3) one gets

$$
\dddot{\sigma}=\bar{u}+\Delta
$$

\section{A. Assumptions and recalls}

Before designing $\bar{u}$, consider the following assumption on the uncertainty.

Assumption 1: The derivative of the uncertainty $\Delta$ is bounded and maximum bound is known. Also, at $t=0$, $\Delta(0)=0$.

Now, one has to design a $\bar{u}$, in order to get $\sigma=\dot{\sigma}=\ddot{\sigma}=0$ in a predefined time $t_{F}$, in spite of perturbation term $\Delta$. System (5) reads as,

$$
\begin{aligned}
\dot{x} & =A x+B(\bar{u}+\Delta) \\
& =\left[\begin{array}{lll}
0 & 1 & 0 \\
0 & 0 & 1 \\
0 & 0 & 0
\end{array}\right] x+\left[\begin{array}{l}
0 \\
0 \\
1
\end{array}\right](\bar{u}+\Delta)
\end{aligned}
$$

Defining $x=\left[\begin{array}{ccc}\sigma & \dot{\sigma} & \ddot{\sigma}\end{array}\right]^{\top}$. Before proceeding further let us recall some standard definition of third order sliding mode as follows,

Definition 1: [5] Consider the system (6) with sliding variable $\sigma$. Assume that the time derivative of sliding variable $\dot{\sigma}, \ddot{\sigma}$ are continuous function. The manifold defined as $\sum^{3}=\{x \mid \sigma=\dot{\sigma}=\ddot{\sigma}=0\}$ is called "third order sliding set", which is no-empty and is locally an integral set in the
Filippov sense. The motion on this set is called third order sliding mode with respect to the sliding variable $\sigma$.

Definition 2: [5] Consider the system (6) with sliding variable $\sigma$. Assume that the time derivative of sliding variable $\dot{\sigma}, \ddot{\sigma}$ are continuous function. The manifold defined as $\sum_{*}^{3}=\left\{x|| \sigma\left|\leq \mu_{0} \tau^{3},\right| \dot{\sigma}\left|\leq \mu_{1} \tau^{2},\right| \ddot{\sigma} \mid \leq \mu_{2} \tau\right\}$ with $\mu_{0}, \mu_{1}, \mu_{2}>0$ and the $\tau$ sampling time, is called "real third order sliding set", which is no-empty and is locally an integral set in the Filippov sense. The motion on this set is called real third order sliding mode with respect to the sliding variable $\sigma$.

Theorem 1: [22] The system (6) with $\Delta=0$ is stabilized in a finite time $t_{F}$ by a control input $\bar{u}$ defined as

$$
\bar{u}= \begin{cases}-B^{\top} M\left[\begin{array}{lll}
\sigma & \dot{\sigma} & \ddot{\sigma}
\end{array}\right]^{\top}+B^{\top} \delta(t) & \text { for } 0 \leq t \leq t_{F} \\
-B^{\top} M\left[\begin{array}{lll}
\sigma & \dot{\sigma} & \ddot{\sigma}
\end{array}\right]^{\top} & \text { for } t>t_{F}\end{cases}
$$

with $\delta(t)(\delta(0)$ selected in order to satisfy the terminal condition $x\left(t_{F}\right)=0$.) and $M$ defined as

$$
\begin{aligned}
& \dot{\delta}=-\left(A^{\top}-M B B^{\top}\right) \delta \\
& 0=M A+A^{\top} M-M B B^{\top} M+Q
\end{aligned}
$$

The details of closed-loop stability analysis with above control is discussed in the [18], [22].

The controller proposed in the sequel is based on the integral sliding mode concept developed in [17]. This kind of controller is composed of two terms. The term required to cancel the perturbation is discussed in the next subsection by using disturbance observer.

\section{B. Disturbance observer and main result}

The goal of the disturbance observer is to estimate the disturbance early from $t \geq 0$. All the states $x$ of the system are measurable. In observer design, we consider the measured variable as

$$
s=G x \quad \text { with } \quad G B \neq 0, \quad G \in \mathbb{R}^{1 \times 3}
$$

Dynamics for the disturbance observer is defined as

$$
\dot{\hat{s}}=G A x+G B \bar{u}+z, \quad \hat{s}(0)=s(0)
$$

where $z$ is the correction term that is defined in the sequel. The error between $s$ and $\hat{s}$ being defined as $e=s-\hat{s}$ (with $e(0)=0)$ ensuring $s=\hat{s}$, its dynamics reads as

$$
\begin{aligned}
\dot{e} & =\dot{s}-\dot{\hat{s}} \\
& =G A x+G B \bar{u}+G B \Delta-G A x-G B u-z \\
& =G B \Delta-z
\end{aligned}
$$

Define the correction term $z$ as

$$
\begin{aligned}
z & =k_{1}|e|^{\frac{1}{2}} \operatorname{sign}(e)-\nu_{1} \\
\dot{\nu}_{1} & =-k_{2} \operatorname{sign}(e)
\end{aligned}
$$


After substituting $z$ in (10), one gets

$$
\begin{aligned}
& \dot{e}=-k_{1}|e|^{\frac{1}{2}} \operatorname{sign}(e)+\nu \\
& \dot{\nu}=-k_{2} \operatorname{sign}(e)+G B \dot{\Delta}
\end{aligned}
$$

where $\nu=G B \Delta-\nu_{1}$. As mentioned earlier at $t=0$ one has $e(0)=0$ which can be maintained with the following gain conditions [17]

$$
k_{2}>\rho, \quad k_{1}>1.41 \sqrt{k_{2}+\rho}, \quad \rho=|G B \dot{\Delta}|_{\max }
$$

and achieves the second order sliding mode on $e$ in the presence of disturbance. Given that $e=\dot{e}=0$, the exact perturbation can be computed from (10) as $\Delta=(G B)^{-1} z$.

Theorem 2: Consider the system (5) with the vector $B=$ $\left[\begin{array}{lll}0 & 0 & 1\end{array}\right]^{\top}$ and vector $G$ defined such that $G B \neq 0$. Suppose that $\Delta$ fulfills Assumption 1 and $|G B \dot{\Delta}|_{\max }=\rho$ with $\Delta(0)=0$. Define the convergence time $t_{F}$. Then, the continuous control input $\bar{u}=u_{n}+u_{d}$ defined as

$$
u_{n}= \begin{cases}-B^{\top} M\left[\begin{array}{ccc}
\sigma & \dot{\sigma} & \ddot{\sigma}
\end{array}\right]^{\top}+B^{\top} \delta(t) & \text { for } 0 \leq t \leq t_{F} \\
-B^{\top} M\left[\begin{array}{lll}
\sigma & \dot{\sigma} & \ddot{\sigma}
\end{array}\right]^{\top} & \text { for } t>t_{F}\end{cases}
$$

with $\delta(t)(\delta(0)$ selected in order to satisfy the terminal condition $x\left(t_{F}\right)=0$.) and $M$ as

$$
\begin{aligned}
& \dot{\delta}=-\left(A^{\top}-M B B^{\top}\right) \delta \\
& 0=M A+A^{\top} M-M B B^{\top} M+Q
\end{aligned}
$$

and

$$
u_{d}=-(G B)^{-1} z
$$

$z$ being defined from (11), allows the establishment of a third order sliding mode with respect to $\sigma$ i.e for $t \geq t_{F}$, $\sigma=\dot{\sigma}=\ddot{\sigma}=0$.

Proof: Let us rewrite dynamics of system (6)

$$
\dot{x}=A x+B(\bar{u}+\Delta)
$$

After substituting the control input, system (16) reads as

$$
\begin{aligned}
\dot{x} & =A x+B\left(u_{n}+u_{d}+\Delta\right) \\
& =A x+B u_{n}+B\left(u_{d}+\Delta\right) \\
& =A x+B u_{n}+B\left(-(G B)^{-1} z+\Delta\right) \\
& =A x+B u_{n}
\end{aligned}
$$

Using disturbance observer, disturbance is estimated exactly so $-(G B)^{-1} z+\Delta=0$ from $t \geq 0$, which makes dynamics free from the disturbance.

$$
\dot{x}=A x+B u_{n}
$$

The system dynamics now reads as Theorem 1, such kind of system is finite time stable and one can obtained for system (6) $x=0$ at $t=t_{F}$, that means $\sigma=\dot{\sigma}=\ddot{\sigma}=0$. In other words, controller achieves the establishment of a third order sliding mode with respect to $\sigma$ in the predefined finite time $t_{F}$.. This completes the proof.

\section{EXPERIMENT RESULTS}

The proposed controller is implemented using MATLAB/SIMULINK with dSpace DS1104 datacard. The sampling period is selected as $1 \mathrm{~ms}$. To implement proposed controller, main actuator's position, velocity and acceleration are required. The position of the load mass is measured by using position sensor and then by using differentiator, velocity and acceleration are obtained. Dynamics of the electropneumatic system are nonlinear and affected with perturbation actuator. For this latter, a reference trajectory of the perturbation is selected and tracking is achieved with a PID controller provided by the company that builds the set-up. For simulations and experiments, two different reference trajectories are considered as $y_{\text {ref }}=0.04 \sin (t)$ and $y_{\text {ref }}=0.04$. The external disturbance trajectory is selected as $F_{\text {ext }}=500 \sin (0.5 t)$ (Figure 2). Using an integral sliding mode control approach, the control input is designed, i.e nominal control $u_{n}$ and the disturbance rejection control $u_{d}$. Design of a nominal control with $t_{F}=2 \mathrm{sec}^{1}$ is discussed below. The nominal control reads as

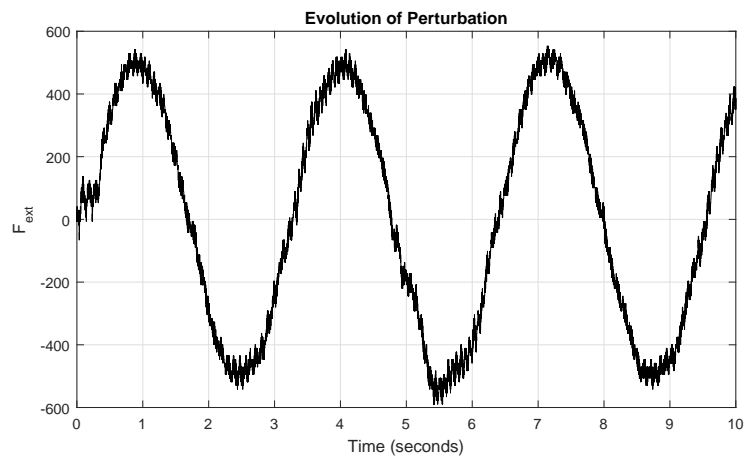

(a)

Fig. 2. External perturbation trajectory obtained using perturbation actuator

$$
u_{n}= \begin{cases}-B^{\top} M x(t)+B^{\top} \delta(t) & \text { for } 0 \leq t \leq t_{F} \\ -B^{\top} M x(t) & \text { for } t>t_{F}\end{cases}
$$

with the matrix $M$ derived from the Riccati's equation, by stating

$$
Q=\left[\begin{array}{lll}
1 & 0 & 0 \\
0 & 1 & 0 \\
0 & 0 & 1
\end{array}\right]
$$

One gets

$$
M=\left[\begin{array}{lll}
2.414 & 2.414 & 1.000 \\
2.414 & 4.828 & 2.414 \\
1.000 & 2.414 & 2.414
\end{array}\right]
$$

The initial condition of the state for sinusoidal tracking is $x(0)=\left[\begin{array}{lll}0 & -0.04 & 0\end{array}\right]^{\top}$ and for constant tracking is $x(0)=\left[\begin{array}{lll}-0.04 & 0 & 0\end{array}\right]^{\top}$. Given $x(0)$ and $t_{F}=2 \mathrm{sec}$,

\footnotetext{
${ }^{1}$ In the sequel, several values of $t_{F}$ will be considered. For each value of $t_{F}$ initial condition of $\delta$ has to be obtained.
} 
initial condition of $\delta(t)$ function is calculated by using the gramper function (For details, see [18], [23] ) i.e.

$$
\begin{aligned}
\delta(0)_{\text {Sinusoidal Reference }} & =\left[\begin{array}{l}
0.9079 \\
0.8989 \\
0.2791
\end{array}\right] \\
\delta(0)_{\text {Constant Reference }} & =\left[\begin{array}{l}
0.9485 \\
0.9079 \\
0.2709
\end{array}\right]
\end{aligned}
$$

The gains of the correction term used in the observer that estimates the disturbance are selected as $k_{1}=700$ and $k_{2}=$ 1000 (see section III). The matrix $G$ is selected as $G=B^{\top}$.

\section{A. Discussions}

During the experimentations to show the effectiveness of the proposed controller, different predefined convergence time $t_{F}$ have been chosen for the tracking of piston position in the presence of external force generated by perturbation actuator. As mentioned in the earlier discussion, two different reference trajectories are considered.

The obtained results are compared with existing method [18]. In Figures 3 (a) and (c), tracking of piston position is depicted with different $t_{F}$ with sinusoidal and constant references. It is clear from Figures 3 (a) and (c) that the proposed controller has achieved the objective in spite of external force. In Figures 3 (b) and (d), same tracking is presented with the controller developed in [18]. The tracking performance are quite similar even if the accuracy is slightly better with the proposed controller. In fact, the main difference (and the main advantage of the proposed method) appears in the control inputs, as displayed by Figures 4 (a) and (b) (only $t_{F}=2 \mathrm{sec}$ is considered). The required control inputs to achieve the control objective are clearly more affected by chattering with control designed from [18]. This kind of signal can be damageable for the servo-distributor. The proposed controller clearly offers a better solution. The pressures in the chamber $N$ are depicted in Figure 4 (c) and (d).

\section{CONCLUSION}

This paper presents practical implementation of a robust third order sliding mode controller for an electropneumatic actuator. The controller guarantees position tracking of the piston at exactly a predefined time $t_{F}$ and this convergence time is chosen in advance. The controller robustness is presented with respect to external disturbances. Implementation results on an experimental setup shows the effectiveness of the controller versus an other third order sliding mode existing control method. It is observed that the proposed control uses less energy to obtain similar performances.

\section{REFERENCES}

[1] X. Brun, S. Sesmat, D. Thomasset, and S. Scavarda, "A comparative study between two control laws of an electropneumatic actuator," in European Control Conference, ECC, Karlsruhe, Germany, 1999, pp. 2967-2974.

[2] M. Bouri and D. Thomasset, "Sliding control of an electropneumatic actuator using an integral switching surface," IEEE Transactions on Control Systems Technology, vol. 9, no. 2, pp. 368-375, 2001.
[3] S. Laghrouche, M. Smaoui, F. Plestan, and X. Brun, "Higher order sliding mode control based on optimal approach of an electropneumatic actuator," International Journal of Control, vol. 79, no. 2, pp. 119-131, 2006.

[4] V. I. Utkin, Sliding modes in control and optimization. Springer, 1992.

[5] Y. Shtessel, C. Edwards, L. Fridman, and A. Levant, Sliding mode control and observation. Springer, New York, USA, 2014.

[6] C. Edwards and S. Spurgeon, Sliding mode control: theory and applications. Taylor and Francis, London, UK, 1998.

[7] A. Levant, "Sliding order and sliding accuracy in sliding mode control," International Journal of Control, vol. 58, no. 6, pp. 1247$1263,1993$.

[8] _-, "Higher-order sliding modes, differentiation and outputfeedback control," International Journal of Control, vol. 76, no. 9-10, pp. 924-941, 2003.

[9] - "Homogeneity approach to high-order sliding mode design," Automatica, vol. 41, no. 5, pp. 823-830, 2005.

[10] J. A. Moreno and M. Osorio, "Strict lyapunov functions for the supertwisting algorithm," IEEE Transactions on Automatic Control, vol. 57, no. 4, pp. 1035-1040, 2012.

[11] J. Davila, L. Fridman, and A. Levant, "Second-order sliding-mode observer for mechanical systems," IEEE Transactions on Automatic Control, vol. 50, no. 11, pp. 1785-1789, 2005.

[12] Y. Shtessel, M. Taleb, and F. Plestan, "A novel adaptive-gain supertwisting sliding mode controller: Methodology and application," Automatica, vol. 48, no. 5, pp. 759-769, 2012.

[13] M. Taleb, A. Levant, and F. Plestan, "Pneumatic actuator control: Solution based on adaptive twisting and experimentation," Control Engineering Practice, vol. 21, no. 5, pp. 727-736, 2013.

[14] X. Yan, F. Plestan, and M. Primot, "A new third-order slidingmode controller-application to an electropneumatic actuator," IEEE Transactions on Control Systems Technology, vol. 25, no. 2, pp. 744 751, 2017.

[15] X. Yan, A. Estrada, and F. Plestan, "Adaptive pulse output feedback controller based on second-order sliding mode: Methodology and application," IEEE Transactions on Control Systems Technology, vol. 24, no. 6, pp. 2233-2240, 2016.

[16] A. Estrada and F. Plestan, "Second order sliding mode output feedback control with switching gains application to the control of a pneumatic actuator," Journal of the Franklin Institute, vol. 351, no. 4, pp. 23352355, 2014.

[17] A. Chalanga and F. Plestan, "Finite time stabilization of an uncertain chain of integrators by integral sliding mode approach," in To appear in 20th IFAC World Congress, Toulouse, France, 2017.

[18] S. Laghrouche, F. Plestan, and A. Glumineau, "Higher order sliding mode control based on integral sliding mode," Automatica, vol. 43, no. 3, pp. 531-537, 2007.

[19] A. Girin, F. Plestan, X. Brun, and A. Glumineau, "A 3rd order sliding mode controller based on integral sliding mode for an electropneumatic system," in 45th IEEE Conference on Decision and Control, CDC, San Diego, California, USA, 2006, pp. 1617-1622.

[20] A. Girin, X. Brun, and F. Plestan, "A new test bench of electropneumatic system for aeronautics application," in The Tenth Scandinavian International Conference On Fluid Power, SICFP'07, Tampere, Finland.

[21] F. Plestan, Y. Shtessel, V. Bregeault, and A. Poznyak, "Sliding mode control with gain adaptation application to an electropneumatic actuator," Control Engineering Practice, vol. 21, no. 5, pp. 679-688, 2013.

[22] Z. Rekasius, "An alternate approach to the fixed terminal point regulator problem," IEEE Transactions on Automatic Control, vol. 9 , no. 3, pp. 290-292, 1964.

[23] P. de Larminat, Contrôle d'état standard. Hermès Science Publications, 2000. 


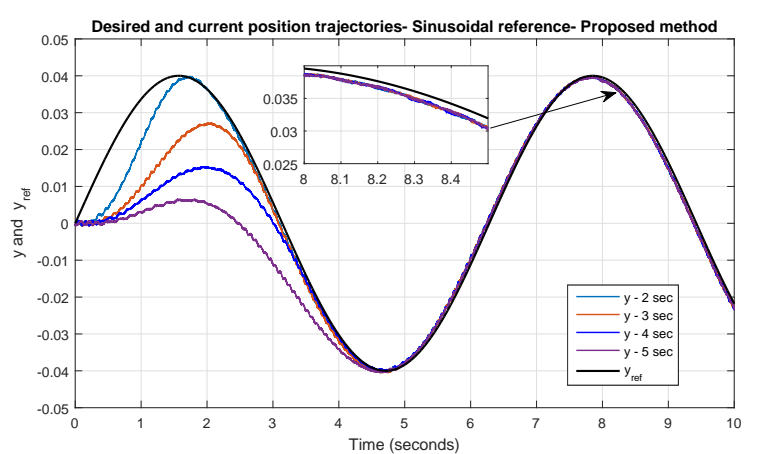

(a)

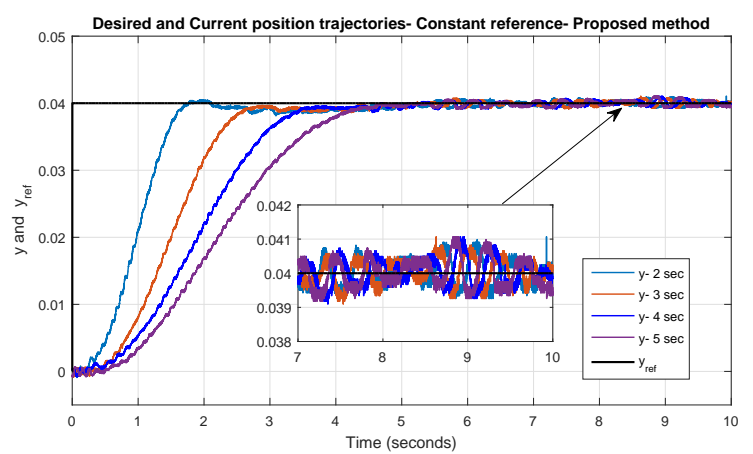

(c)

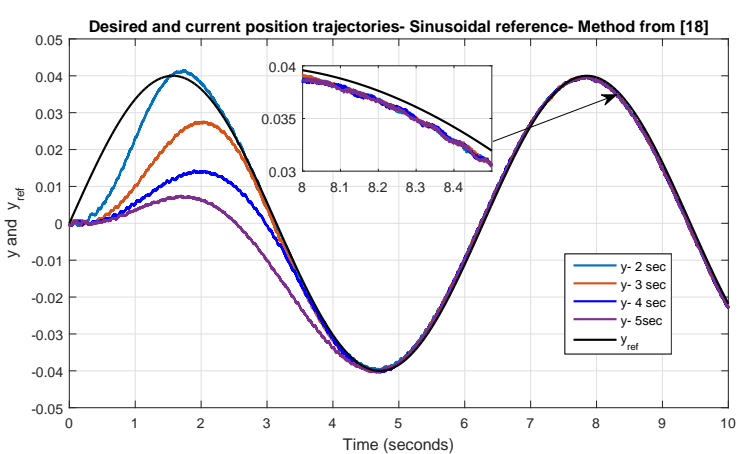

(b)

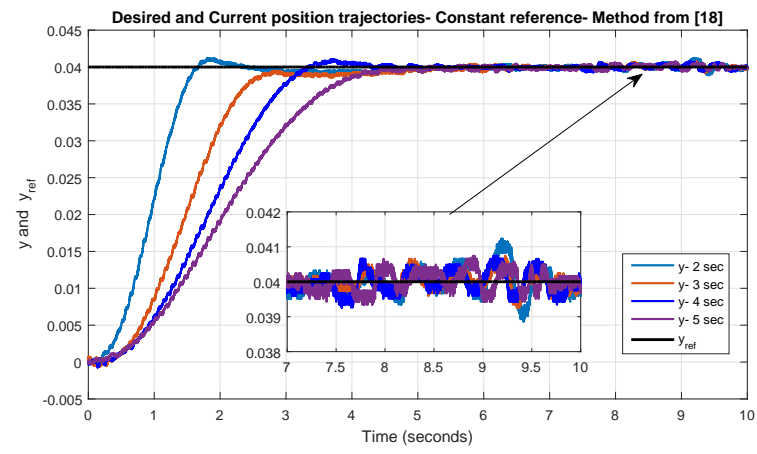

(d)

Fig. 3. Position $y(\mathrm{~m})$ versus time $(\mathrm{sec})$ several predefined times $\left(t_{F}=2,3,4,5 \mathrm{sec}\right)$ : Sinusoidal and Constant Reference Tracking: Left. Proposed method. Right. Method from [18]. 

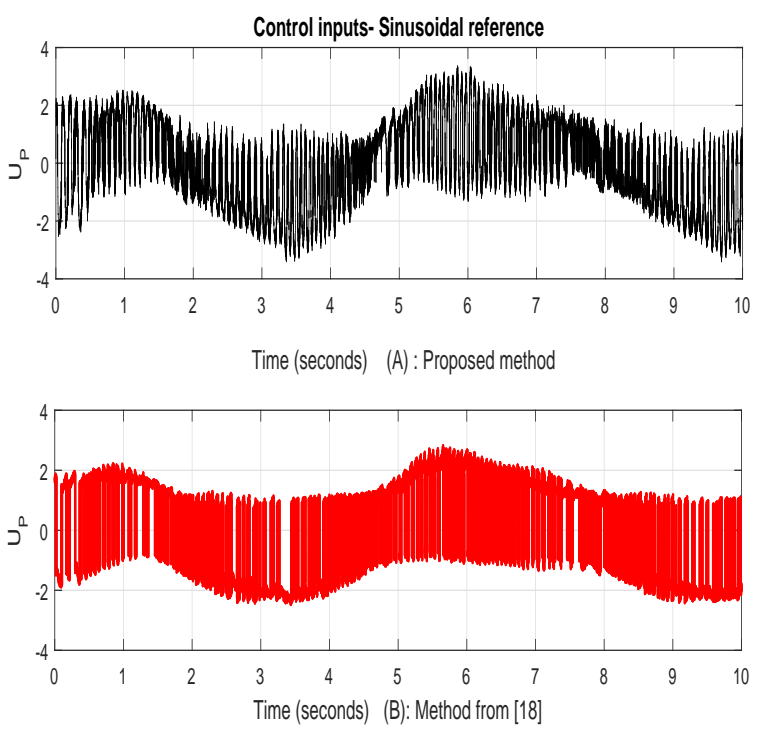

(a)
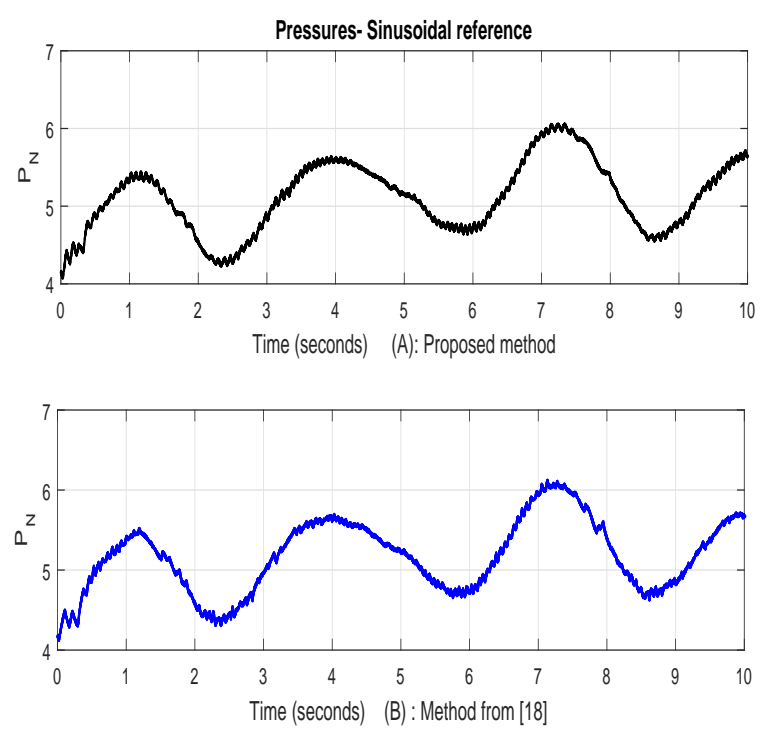

(c)
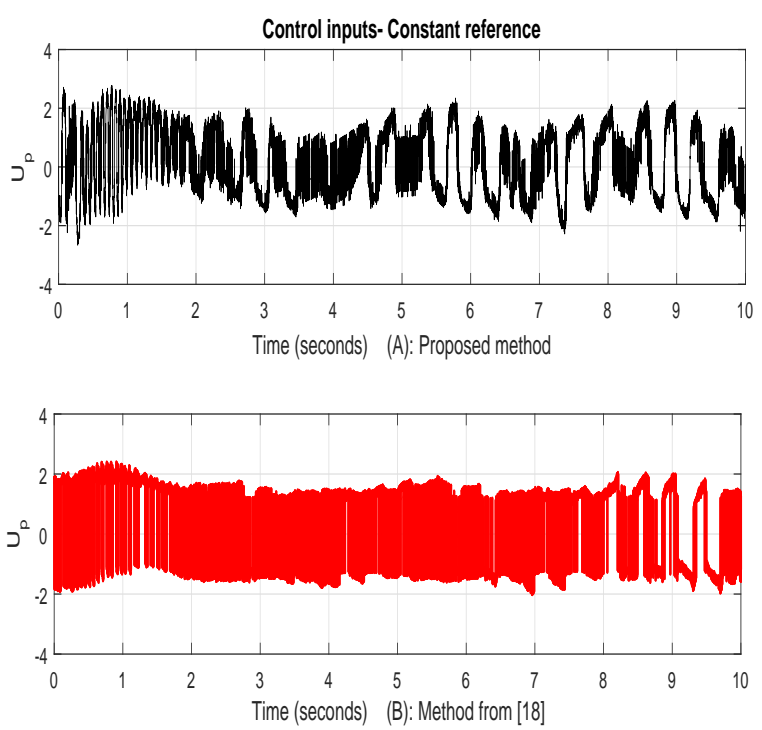

(b)
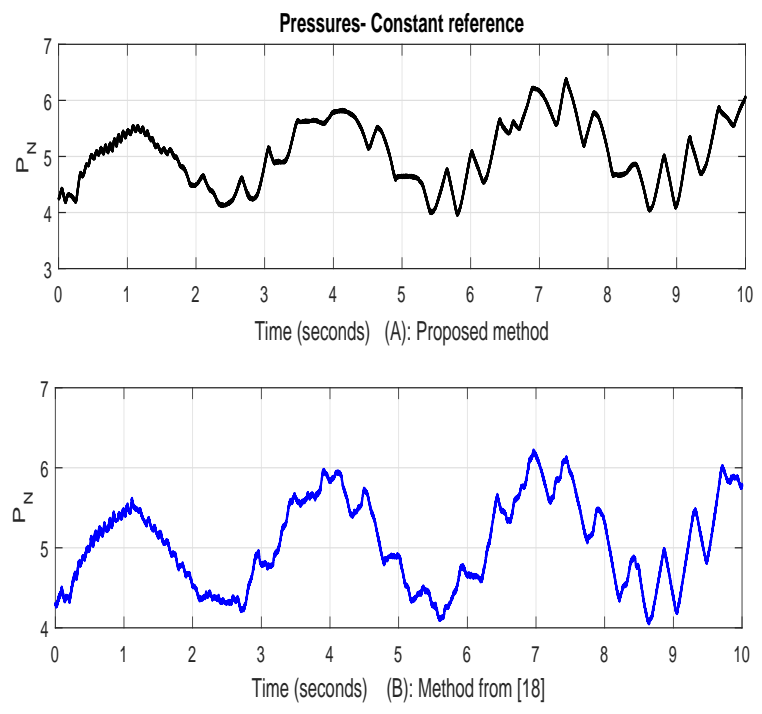

(d)

Fig. 4. Sinusoidal and Constant Reference Tracking $\left(t_{F}=2 \mathrm{sec}\right)$. Plots (a) and (b)- Control input $u_{P}(V)$ versus time (sec), Top. Proposed method. Bottom. Method from. [18], (c) and (d)- $P_{N}$ (bar) versus time (sec), Top. Proposed method. Bottom. Method from [18]. 